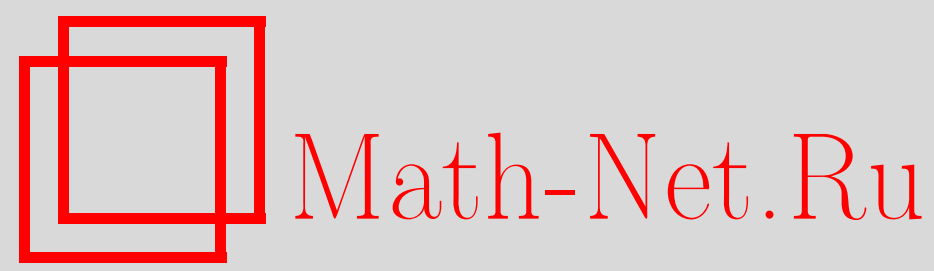

А. В. Грибов, П. А. Золотых, А. В. Михалёв, Построение алгебраической криптосистемы над квазигрупповым кольцом, Матем. вопр. криптогр., 2010, том 1 , выпуск 4, 23-32

DOI: https://doi.org/10.4213/mvk19

Использование Общероссийского математического портала Math-Net.Ru подразумевает, что вы прочитали и согласны с пользовательским соглашением

http://www.mathnet.ru/rus/agreement

Параметры загрузки:

IP : 35.173 .219 .12

26 апреля 2023 г., 16:45:48 


\title{
Построение алгебраической криптосистемы над квазигрупповым кольцом
}

\author{
А. В. Грибов, П. А. Золотых, А. В. Михалёв \\ Московский государственный университет им. М.В.Ломоносова
}

Получено 22.IV.2010

Наиболее распространенными криптосистемами с открытым ключом являются RSA, криптосистема Эль-Гамаля, а также схемы шифрования, в основе которых лежит проблема Диффи - Хеллмана. Мы строим аналогичную криптосистему с помощью неассоциативного квазигруппового кольца. Описаны модификации, увеличивающие стойкость этой схемы относительно возможных атак. Анализируются конкретные неассоциативные алгебраические структуры, пригодные для построения криптосистем.

Ключевые слова: квазигрупповое кольцо, автоморфизмы квазигруппового кольца, криптосистема с открытым ключом

A construction of algebraic cryptosystem over the quasigroup ring

\section{A. V. Gribov, P.A. Zolotykh, A.V. Mikhalev \\ Lomonosov Moscow state university}

Abstract. Nowadays the most popular public key cryptosystems are RSA, the ElGamal cryptosystem and encryption schemes based on the Diffie-Hellman problem. We construct a similar cryptosystem by means of a non-associative structure, namely, quasigroup ring. Some modifications increasing the security of this scheme against possible attacks are described. Several concrete non-associative algebraic structures acceptable for cryptosystem constructions were considered and analyzed also.

Key words: quasigroup ring, automorphism of quasigroup, public-key cryptosystem

Citation: Mathematical Aspects of Cryptography, 2010, vol. 1, no. 4, pp. 23-32 (Russian). 


\section{1. Вводные понятия}

Пусть $K-$ ассоциативное кольцо с единицей, $L-$ лупа (квазигруппа с единицей). Рассмотрим множество $K L$, состоящее из всех формальных сумм вида $\sum_{l \in L} \alpha_{l} \cdot l\left(\alpha_{l} \in K\right)$, в которых конечное число $\alpha_{l}$ отлично от нуля. Два элемента $a, b \in K L$ считаются равными тогда и только тогда, когда $\alpha_{l}=\beta_{l}$ для всех $l \in L$.

На множестве $K L$ определены операции сложения и умножения следующим образом: если $a=\sum_{l \in L} \alpha_{l} \cdot l$ и $b=\sum_{l \in L} \beta_{l} \cdot l$ - элементы $K L$, то

$$
a+b=\sum_{l \in L}\left(\alpha_{l}+\beta_{l}\right) \cdot l, \quad a b=\sum_{l \in L}\left(\sum_{m, h \in L: m h=l} \alpha_{m} \beta_{h}\right) l .
$$

Таким образом, относительно этих операций множество $K L$ является неассоциативным кольцом с единицей. Удобно отождествить $l \in L$ с элементом $1 \cdot l \in K L$, а $\alpha \in K-$ с элементом $\alpha \cdot e$, где $e-$ единица лупы, тогда $K$ и $L$ являются подмножествами в $K L$. Предполагаем, что группы автоморфизмов Aut $K$ и Aut $L$ некоммутативны, причем $\mid$ Aut $K \mid \geq t_{1}$, $\mid$ Aut $L \mid \geq t_{2}$, где $t_{1}$ и $t_{2}$ - параметры безопасности. Также предполагаем, что в $K L$ достаточно элементов с нулевым левым аннулятором.

Рассмотрим следующую задачу. Пусть $R-$ кольцо или лупа, $A-$ некоторое подмножество автоморфизмов в Aut $R, \alpha-$ случайно выбранный элемент из $A$. Предположим, что известно некоторое множество пар $\left(x_{i}, \alpha\left(x_{i}\right)\right), i=1, \ldots, n$, где $x_{i} \in R$. Требуется найти такой автоморфизм $\alpha^{\prime} \in A$, что $\alpha^{\prime}\left(x_{i}\right)=\alpha\left(x_{i}\right), i=1, \ldots, n$. Обозначим эту задачу через $\Omega_{n}(A, R)$.

Заметим, что при отсутствии существенной информации о множествах $A$ и $R$ задача $\Omega_{n}(A, R)$ является вычислительно трудной, поскольку она разрешима только полным перебором всех элементов множества $A$ и для каждого выбранного $\alpha^{\prime} \in A-$ проверкой условия $\alpha^{\prime}\left(x_{i}\right)=\alpha\left(x_{i}\right), i=1, \ldots, n$.

\section{2. Построение алгебраической криптосистемы над кольцом и лупой}

Построим на задаче $\Omega_{n}$ криптосистему.

Участник $A$ :

1. Конструирует такой автоморфизм $\sigma \in$ Aut $K$, что $|\sigma| \geq t_{3}, \sigma$ имеет нетривиальный централизатор $C(\sigma)$ и $|C(\sigma) \backslash\langle\sigma\rangle| \geq t_{4}$, где $t_{3}, t_{4}-$ параметры безопасности. 
2. Конструирует такой автоморфизм $\eta \in$ Aut $L$, что $|\eta| \geq t_{5}, \eta$ имеет нетривиальный централизатор $C(\eta)$ и $|C(\eta) \backslash\langle\eta\rangle| \geq t_{6}$, где $t_{5}, t_{6}$ - параметры безопасности.

3. Случайно выбирает автоморфизм $\tau \in C(\sigma) \backslash\langle\sigma\rangle$.

4. Случайно выбирает автоморфизм $\omega \in C(\eta) \backslash\langle\eta\rangle$.

5. По $\tau$ и $\omega$ строит автоморфизм $\varphi \in$ Aut $K L$ (назовем его секретным автоморфизмом) так:

$$
\varphi(h)=\tau\left(a_{l_{1}}\right) \omega\left(l_{1}\right)+\cdots+\tau\left(a_{l_{n}}\right) \omega\left(l_{n}\right)
$$

для любого $h \in K L$ вида $h=a_{l_{1}} l_{1}+\cdots+a_{l_{n}} l_{n}$, где $L=\left\{l_{1}, \ldots, l_{n}\right\}$, $a_{l_{1}}, \ldots, a_{l_{n}} \in K$.

6. Выбирает элементы $a \in K L, x \in K L$.

7. Вычисляет $\varphi(x)$ и $\varphi(a)$.

В результате получается открытый ключ участника $A$

$$
(\sigma, \eta, x, \varphi(x), a, \varphi(a)) .
$$

Участник $B$ :

1. Выбирает натуральные числа $(i, j, k, l)$.

2. Используя открытый ключ участника $A$, получает пары автоморфизмов $\left(\sigma^{i}, \eta^{j}\right),\left(\sigma^{k}, \eta^{l}\right)$ и по ним строит автоморфизмы $\psi, \chi \in$ Aut $K L$ таким же способом, как и участник $A$, т. е.

$$
\begin{aligned}
& \psi(h)=\sigma^{i}\left(a_{l_{1}}\right) \eta^{j}\left(l_{1}\right)+\cdots+\sigma^{i}\left(a_{l_{n}}\right) \eta^{j}\left(l_{n}\right), \\
& \chi(h)=\sigma^{k}\left(a_{l_{1}}\right) \eta^{l}\left(l_{1}\right)+\cdots+\sigma^{k}\left(a_{l_{n}}\right) \eta^{l}\left(l_{n}\right)
\end{aligned}
$$

для любого $h \in K L$ вида $h=a_{l_{1}} l_{1}+\cdots+a_{l_{n}} l_{n}$. Автоморфизмы $\psi, \chi$ будем называть сеансовыми. Так как $\tau \in C(\sigma) \backslash\langle\sigma\rangle$ и $\omega \in C(\eta) \backslash\langle\eta\rangle$, то автоморфизмы $\tau$ и $\sigma, \omega$ и $\eta$ попарно коммутируют. Поэтому коммутируют и построенные по ним автоморфизмы $\varphi$ и $\psi, \varphi$ и $\chi$.

3. Вычисляет $\chi(a) \cdot \psi(x)$.

4. Вычисляет $\chi(\varphi(a)) \cdot \psi(\varphi(x))$ и левый аннулятор $\operatorname{Ann}(\chi(\varphi(a)) \cdot \psi(\varphi(x)))$.

2010, T. 1, № 4, C. 23-32 
5. Если полученный аннулятор $\operatorname{Ann}(\chi(\varphi(a)) \cdot \psi(\varphi(x)))$ ненулевой, то проводится новый сеанс связи с выбором новых элементов $a$ и $x$ или же выбираются другие сеансовые автоморфизмы.

6. Записывает исходный текст, который надо передать, в виде $m \in K L$ и вычисляет $m \cdot[\chi(\varphi(a)) \cdot \psi(\varphi(x))]$.

7. Отправляет $A$ криптограмму

$$
(\chi(a) \cdot \psi(x), m \cdot[\chi(\varphi(a)) \cdot \psi(\varphi(x))]) .
$$

Получив криптограмму, участник $A$ расшифровывает ее:

1. Используя секретный автоморфизм $\varphi$, вычисляет $\varphi(\chi(a) \cdot \psi(x))$.

2. Расшифровывает посланный текст пользуясь тем, что $\chi, \psi$ и $\varphi$ коммутируют, поскольку сеансовые автоморфизмы $\psi, \chi$ построены на степенях выбранных автоморфизмов $\sigma, \eta$, а секретный автоморфизм $\varphi$ построен с помощью элементов из централизаторов $\sigma, \eta$. Таким образом, участник А знает $m \cdot[\varphi(\chi(a) \cdot \psi(x))]$ и $\varphi(\chi(a) \cdot \psi(x))$; следовательно, для получения сообщения $m$ ему достаточно решить линейную систему с коэффициентами из кольца $K$. Для этого элемент $\chi(\varphi(a)) \cdot \psi(\varphi(x))$ выбирается с нулевым левым аннулятором. Это нетрудно сделать, если в качестве $K$ взять конечномерную алгебру над полем. Можно в качестве $K$ брать и другие кольца, главное, чтобы можно было решать систему линейных уравнений с коэффициентами из этого кольца.

\section{3. Анализ атак на криптосистему}

Рассмотрим некоторые атаки на криптосистему.

\section{1. Атака только с криптограммой.}

Пусть криптоаналитик располагает открытым ключом участника $A$ и криптограммой. Перед ним стоит следующая задача: по известным парам $(a, \varphi(a)),(x, \varphi(x))$ найти такой $\alpha \in \operatorname{Aut} K L$, индуцированный автоморфизмами $\left(\sigma^{\prime}, \eta^{\prime}\right)$, что $\varphi(a)=\alpha(a), \varphi(x)=\alpha(x)$. К тому же необходимо, чтобы $\sigma^{\prime} \in C(\sigma) \backslash\langle\sigma\rangle$, a $\eta^{\prime} \in C(\eta) \backslash\langle\eta\rangle$.

Построим $\alpha$. Положим $\alpha(a):=\varphi(a), \alpha(x):=\varphi(x)$. Тогда определяются

$$
\alpha(a x)=\alpha(a) \cdot \alpha(x):=\varphi(a) \cdot \varphi(x) \quad \text { и } \quad \alpha(x a)=\alpha(x) \cdot \alpha(a):=\varphi(x) \cdot \varphi(a) .
$$

Но доопределить действие $\alpha$ на элемент $\chi(a) \cdot \psi(x)$ можно лишь перебором его образа с последующей проверкой того, что $\sigma^{\prime} \in C(\sigma) \backslash\langle\sigma\rangle$, а $\eta^{\prime} \in C(\eta) \backslash\langle\eta\rangle$. 
Это вычислительно не легче перебора всех автоморфизмов, индуцированных парами $\left(\sigma^{\prime}, \eta^{\prime}\right) \in(C(\sigma) \backslash\langle\sigma\rangle) \times(C(\eta) \backslash\langle\eta\rangle)$, удовлетворяющих начальным условиям $\alpha(a)=\varphi(a)$ и $\alpha(x)=\varphi(x)$. В итоге получаем задачу $\Omega_{2}(Y, K L)$, где $Y$ - это множество автоморфизмов $K L$, полученных с помощью пар $\left(\sigma^{\prime}, \eta^{\prime}\right) \in[(C(\sigma) \backslash\langle\sigma\rangle) \times(C(\eta) \backslash\langle\eta\rangle)]$.

Для оценки сложности вскрытия криптосистемы злоумышленниками будем использовать отношение объема передаваемой информации ко всему объему, из которого она была выбрана, т. е. $\frac{2}{t_{4} \cdot t_{6}}$. Поэтому при надлежащем выборе параметров безопасности данная задача является вычислительно трудной.

\section{2. Атака на сеансовые автоморфизмы $\psi$ и $\chi$.}

Другой способ атаки - найти автоморфизмы $\psi$ и $\chi$, а затем решить относительно $m$ уравнение $m \cdot[\chi(\varphi(a)) \cdot \psi(\varphi(x))]=h$, где $h$ известен из криптограммы. Пусть $\psi$ был построен с помощью автоморфизмов $\left(\sigma_{1}, \eta_{1}\right)$, а автоморфизм $\chi-\mathrm{c}$ помощью $\left(\sigma_{2}, \eta_{2}\right)$. Чтобы найти $\psi$ и $\chi$, криптоаналитику придется перебрать пары $\left(\sigma_{1}, \eta_{1}\right) \in(\langle\sigma\rangle,\langle\eta\rangle)$ и $\left(\sigma_{2}, \eta_{2}\right) \in(\langle\sigma\rangle,\langle\eta\rangle)$ с последующей проверкой условия $\chi(a) \psi(x)=h_{1}$, где $h_{1}$ известен из криптограммы. Следовательно, определенная выше сложность атаки характеризуется величиной $\frac{1}{t_{3}^{2} \cdot t_{5}^{2}}$. Опять видим, что при правильном выборе соответствующих параметров безопасности эта задача является вычислительно трудной.

Поясним, почему для построения сеансового автоморфизма рассматривался не один автоморфизм, а произведение $\chi(a) \psi(x)$. В случае единственного автоморфизма открытый ключ участника - это $(\sigma, \eta, x, \varphi(x))$, а криптограмма $-(\psi(x), m \cdot \psi(\varphi(x))$. В этом случае стали бы возможны следующие атаки.

а) Атака на сеансовый автоморфизм. Пусть автоморфизм $\psi$, как и раньше, построен с помощью $\left(\sigma^{i}, \eta^{j}\right)$. Для нахождения $\sigma^{i}$ можно учесть тот факт, что любой автоморфизм лупы отображает единицу в единицу. Тогда из коэффициентов при единице лупы $L$ для $x$ и $\psi(x)$ получаем пару элементов кольца $K$ вида $\left(a_{e}, \sigma^{i}\left(a_{e}\right)\right)$; аналогичные рассуждения верны и для коэффициентов при единице кольца $K$. Таким образом, криптоаналитик получает дополнительную информацию о сеансовом автоморфизме $\psi$. В случае выбора произведения двух сеансовых автоморфизмов такая атака невозможна, так как коэффициент при единице у элемента $\psi(x) \in K L$ равен $\sum_{l h=e} \alpha_{l} \beta_{h}$, где $l, h \in L$, а это не позволяет получить пару вида $\left(a_{e}, \sigma^{i}\left(a_{e}\right)\right)$.

б) Атака на автоморфизм ч с известным исходным текстом. Криптоаналитик вычисляет $x \cdot \psi(x)=y$. Тогда $\varphi(x) \cdot \psi(\varphi(x))=\varphi(x \cdot \psi(x))$. Следовательно, у криптоаналитика имеется как пара $(x, \varphi(x))$, так и пара $(y, \varphi(y))$. 
В случае выбора произведения двух сеансовых автоморфизмов такая атака существенно ослаблена, так как не удастся получить вторую пару подобным образом, и придется восстанавливать $\varphi$ по одной паре.

Таким образом, выбор произведения двух сеансовых автоморфизмов в целом оправдан.

\section{3. Атака с выбранным исходным текстом.}

Эта атака основана на попытке криптоаналитика получить $\chi(\varphi(a)) \psi(\varphi(x)) \in K L \quad$ с п последующим решением уравнения $m \times$ $\times \chi(\varphi(a)) \psi(\varphi(x))$ относительно $m$ посредством нового сеанса связи с участником В в качестве участника А. Даже если участник В повторяет тот же исходный текст $m$, то он должен сконструировать новые сеансовые автоморфизмы $\psi^{\prime} \neq \psi$ и $\chi^{\prime} \neq \chi$. Поэтому криптоаналитик получит не $m \cdot \chi(\varphi(a)) \psi(\varphi(x))$, а $m \cdot \chi^{\prime}(\varphi(a)) \psi^{\prime}(\varphi(x))$. И даже если он решит новое уравнение относительно $\chi^{\prime}(\varphi(a)) \psi^{\prime}(\varphi(x))$, никакой новой информации относительно $\chi(\varphi(a)) \psi(\varphi(x))$ он не получит.

\section{4. Поиск подходящих для шифрования колец и луп}

Рассмотрим некоторые структуры, подходящие для построения криптосистемы. В качестве лупы будем брать прямое произведение нескольких групп и луп, варьируя их количество для подбора параметров безопасности.

Для построения примера рассмотрим следующую неассоциативную лупу Муфанг $M$ порядка 16 . Приведем ее таблицу Кэли:

$\begin{array}{cccccccccccccccc}1 & 2 & 3 & 4 & 5 & 6 & 7 & 8 & 9 & 10 & 11 & 12 & 13 & 14 & 15 & 16 \\ 2 & 4 & 8 & 6 & 3 & 1 & 5 & 7 & 14 & 9 & 16 & 10 & 11 & 12 & 13 & 15 \\ 3 & 5 & 4 & 7 & 6 & 8 & 1 & 2 & 15 & 13 & 9 & 11 & 14 & 16 & 12 & 10 \\ 4 & 6 & 7 & 1 & 8 & 2 & 3 & 5 & 12 & 14 & 15 & 9 & 16 & 10 & 11 & 13 \\ 5 & 7 & 2 & 8 & 4 & 3 & 6 & 1 & 13 & 11 & 14 & 16 & 12 & 15 & 10 & 9 \\ 6 & 1 & 5 & 2 & 7 & 4 & 8 & 3 & 10 & 12 & 13 & 14 & 15 & 9 & 16 & 11 \\ 7 & 8 & 1 & 3 & 2 & 5 & 4 & 6 & 11 & 16 & 12 & 15 & 10 & 13 & 9 & 14 \\ 8 & 3 & 6 & 5 & 1 & 7 & 2 & 4 & 16 & 15 & 10 & 13 & 9 & 11 & 14 & 12 \\ 9 & 10 & 11 & 12 & 16 & 14 & 15 & 13 & 4 & 6 & 7 & 1 & 5 & 2 & 3 & 8 \\ 10 & 12 & 16 & 14 & 15 & 9 & 13 & 11 & 2 & 4 & 5 & 6 & 3 & 1 & 8 & 7 \\ 11 & 13 & 12 & 15 & 10 & 16 & 9 & 14 & 3 & 8 & 4 & 7 & 6 & 5 & 1 & 2 \\ 12 & 14 & 15 & 9 & 13 & 10 & 11 & 16 & 1 & 2 & 3 & 4 & 8 & 6 & 7 & 5 \\ 13 & 15 & 10 & 16 & 9 & 11 & 14 & 12 & 8 & 7 & 2 & 5 & 4 & 3 & 6 & 1 \\ 14 & 9 & 13 & 10 & 11 & 12 & 16 & 15 & 6 & 1 & 8 & 2 & 7 & 4 & 5 & 3 \\ 15 & 16 & 9 & 11 & 14 & 13 & 12 & 10 & 7 & 5 & 1 & 3 & 2 & 8 & 4 & 6 \\ 16 & 11 & 14 & 13 & 12 & 15 & 10 & 9 & 5 & 3 & 6 & 8 & 1 & 7 & 2 & 4\end{array}$


Отметим, что $\mid$ Aut $M \mid=1344$, причем система порождающих элементов в группе Aut $M$ (если ее представить в виде подгруппы группы перестановок) состоит из двух элементов:

$$
\begin{gathered}
a=(2,3)(5,8)(6,7)(10,11)(13,16)(14,15), \\
b=(2,14,13,15,8,9,3)(5,12,7,6,10,16,11) .
\end{gathered}
$$

Заметим, что $|a|=2,|b|=7$, a $|C(a)|=16,|C(b)|=7$.

Усложним структуру. Теперь представим $S=\operatorname{Aut}(M \times M),|S|=$ $=231211008$ в виде подгруппы группы перестановок. Система порождающих:

$a=(129,145,177,209)(130,146,178,210)(131,147,179,211)(132,148,180,212)$

$(133,149,181,213)(134,150,182,214)(135,151,183,215)(136,152,184,216)$

$(137,153,185,217)(138,154,186,218)(139,155,187,219)(140,156,188,220)$

$(141,157,189,221)(142,158,190,222)(143,159,191,223)(144,160,192,224)$

$(161,241,225,193)(162,242,226,194)(163,243,227,195)(164,244,228,196)$

$(165,245,229,197)(166,246,230,198)(167,247,231,199)(168,248,232,200)$

$(169,249,233,201)(170,250,234,202)(171,251,235,203)(172,252,236,204)$

$(173,253,237,205)(174,254,238,206)(175,255,239,207)(176,256,240,208)$,

$b=(17,132,36,20,129,33)(18,134,38,22,130,34)(19,135,39,23,131,35)$

$(21,136,40,24,133,37)(25,140,44,28,137,41)(26,142,46,30,138,42)$

$(27,143,47,31,139,43)(29,144,48,32,141,45)(65,212,161)(66,214,162)$

$(67,215,163)(68,209,164)(69,216,165)(70,210,166)(71,211,167)(72,213,168)$

$(73,220,169)(74,222,170)(75,223,171)(76,217,172)(77,224,173)(78,218,174)$

$(79,219,175)(80,221,176)(81,180,100,84,177,97)(82,182,102,86,178,98)$

$(83,183,103,87,179,99)(85,184,104,88,181,101)(89,188,108,92,185,105)$

$(90,190,110,94,186,106)(91,191,111,95,187,107)(93,192,112,96,189,109)$, $c=(113,148,225)(114,150,226)(115,151,227)(116,145,228)(117,152,229)$

$(118,146,230)(119,147,231)(120,149,232)(121,156,233)(122,158,234)$

$(123,159,235)(124,153,236)(125,160,237)(126,154,238)(127,155,239)$

$(128,157,240)(193,196)(194,198)(195,199)(197,200)(201,204)(202,206)$

$(203,207)(205,208)(241,244)(242,246)(243,247)(245,248)(249,252)$

$(250,254)(251,255)(253,256)(2,17)(3,33)(4,49)(5,65)(6,81)(7,97)$

$(8,113)(9,129)(10,145)(11,161)(12,177)(13,193)(14,209)(15,225)$

$(16,241)(19,34)(20,50)(21,66)(22,82)(23,98)(24,114)(25,130)(26,146)$

$(27,162)(28,178)(29,194)(30,210)(31,226)(32,242)(36,51)(37,67)(38,83)$

$(39,99)(40,115)(41,131)(42,147)(43,163)(44,179)(45,195)(46,211)$

2010, T. 1, № 4, C. 23-32 


$$
\begin{gathered}
(47,227)(48,243)(53,68)(54,84)(55,100)(56,116)(57,132)(58,148)(59,164) \\
(60,180)(61,196)(62,212)(63,228)(64,244)(70,85)(71,101)(72,117) \\
(73,133)(74,149)(75,165)(76,181)(77,197)(78,213)(79,229)(80,245) \\
(87,102)(88,118)(89,134)(90,150)(91,166)(92,182)(93,198)(94,214) \\
(95,230)(96,246)(104,119)(105,135)(106,151)(107,167)(108,183) \\
(109,199)(110,215)(111,231)(112,247)(121,136)(122,152)(123,168) \\
(124,184)(125,200)(126,216)(127,232)(128,248)(138,153)(139,169) \\
(140,185)(141,201)(142,217)(143,233)(144,249)(155,170)(156,186) \\
(157,202)(158,218)(159,234)(160,250)(172,187)(173,203)(174,219) \\
(175,235)(176,251)(189,204)(190,220)(191,236)(192,252)(206,221) \\
(207,237)(208,253)(223,238)(224,254)(240,255),
\end{gathered}
$$

причем $|a|=4,|b|=6,|c|=2$.

Рассмотрим централизаторы этих элементов: $|C(a)|=1376256$, $|C(b)|=129024,|C(c)|=21504$. Как видим, даже на структурах такого маленького порядка получаются достаточно большие централизаторы. Итак, если включить несколько таких луп $M$ в прямое произведение, то это будет лупа с большим количеством автоморфизмов, среди которых есть элементы с большими централизаторами.

Чтобы увеличить порядок самих автоморфизмов, сделаем следующее. Рассмотрим циклическую группу $H=\langle a\rangle$, порожденную элементом $a$. Как известно, $\mid$ Aut $H \mid=\varphi(a)$, где $\varphi-$ функция Эйлера. Мы всегда можем подобрать порядок $a$ таким образом, чтобы в Aut $H$ существовали автоморфизмы большого порядка. Итак, если взять лупу вида $S=M \times M \times \ldots \times M \times H \times$ $\times H \times \ldots \times H$, то в группе ее автоморфизмов будет достаточно много элементов, подходящих для построения криптосистемы.

В качестве кольца рассмотрим $K=M_{2}\left(Z_{5}\right)$ - кольцо квадратных матриц второго порядка над $Z_{5}$. Пусть $A=\left(\begin{array}{ll}1 & 4 \\ 0 & 1\end{array}\right) \in K^{*}$ и $\sigma$ - внутренний автоморфизм кольца $K$, индуцированный матрицей $A$. Понятно, что степени автоморфизма индуцированы соответствующими степенями матрицы $A$, а значит, $|\sigma|=5$. Для простоты будем рассматривать централизатор $C^{\prime}(\sigma)$ автоморфизма $\sigma$ в группе Int $K$ внутренних автоморфизмов кольца $K$, и тогда $\left|C^{\prime}(\sigma)\right|=20$.

Для построения квазигруппового кольца возьмем, например, $K=$ $=M_{2}\left(Z_{5}\right)$ и $S=M \times M$. Так как $|K|=625,|S|=256$, то $|K S|=625^{256}>$ $>2^{2304}$. Заметим, что и автоморфизмов довольно много:

$$
\mid \text { Aut } K|>| \operatorname{Int} K|=| G L_{2}\left(Z_{5}\right)|=480, \quad| \text { Aut } S \mid=231211008 \text {, }
$$


а число автоморфизмов $K S$ равно $\mid$ Aut $K|\cdot|$ Aut $S \mid=11098128384$. Если брать в качестве $S$ прямое произведение большего количества $M$ и $H$, то получим большое число автоморфизмов при сравнительно небольшом размере структуры $K S$.

\section{5. Некоторые модификации криптосистемы}

Для повышения устойчивости к атакам описанную криптосистему можно модифицировать следующим образом: записывая исходный текст $m$ для передачи, можно домножать его не только справа, но и слева на заданные автоморфизмами лупы и кольца элементы (аналогично сконструировав дополнительные автоморфизмы и домножив $m$ ). Зашифрованный текст будет иметь вид

$$
\chi^{\prime}\left(\varphi^{\prime}\left(a^{\prime}\right)\right) \cdot \psi^{\prime}\left(\varphi^{\prime}\left(x^{\prime}\right)\right) \cdot[m \cdot \chi(\varphi(a)) \cdot \psi(\varphi(x))] .
$$

Конечно, здесь предполагается, что у $\chi^{\prime}\left(\varphi^{\prime}\left(a^{\prime}\right)\right) \cdot \psi^{\prime}\left(\varphi^{\prime}\left(x^{\prime}\right)\right)$ есть нулевой правый аннулятор.

Эта модификация значительно ослабит возможности атаки с известным исходным текстом, так как, даже зная $m$, мы не сможем решить уравнение относительно него.

Другая модификация заключается в следующем. Вместо одного элемента $a$ можно взять $r$ штук: $a_{1}, \ldots, a_{r}$, и аналогично построить для них автоморфизмы $\chi_{1}, \ldots, \chi_{r}$. Криптограмма для $A$ в таком случае будет иметь вид

$$
\left(\chi_{1}\left(a_{1}\right) \cdot \ldots \cdot \chi_{r}\left(a_{r}\right) \cdot \psi(x), m \cdot\left[\chi_{1}\left(\varphi\left(a_{1}\right)\right) \cdot \ldots \cdot \chi_{r}\left(\varphi\left(a_{r}\right)\right) \cdot \psi(\varphi(x))\right]\right) .
$$

В этой криптограмме в элементах $\chi_{1}\left(\varphi\left(a_{1}\right)\right) \cdot \ldots \cdot \chi_{r}\left(\varphi\left(a_{r}\right)\right) \cdot \psi(\varphi(x))$ и $\chi_{1}\left(a_{1}\right) \cdot \ldots \cdot \chi_{r}\left(a_{r}\right) \cdot \psi(x)$ намеренно не расставлены скобки, так как нам не важно, в каком порядке перемножать. Главное, чтобы полученный элемент имел нулевой левый аннулятор. Тогда участник А сможет просто решить систему уравнений для нахождения обратного. Для взломщика же усложнится атака на сеансовые автоморфизмы, так как придется перебирать не только степени автоморфизмов, но и всевозможные порядки умножения элементов. Действительно, элементы $a_{1}, \ldots, a_{r}$ и автоморфизмы $\chi_{1}, \ldots, \chi_{r}$ выбраны независимо. Поэтому, атакуя сеансовые автоморфизмы, мы сталкиваемся с $r+1$ задачами $\Omega_{1}(L(\langle\sigma\rangle,\langle\eta\rangle), K L)$, к тому же вынуждены перепробовать всевозможную расстановку скобок. Это существенно усложняет атаку на сеансовые автоморфизмы. С другой стороны, в атаке только с криптограммой мы имеем задачу уже не $\Omega_{2}(L, K L)$, а $\Omega_{r+1}(L, K L)$. Заметим, что 
характеристика сложности атаки на сеансовый автоморфизм будет равна $\frac{1}{t_{3}^{r+1} \cdot t_{5}^{r+1}} \cdot \frac{r !(r+1) !}{(2 r) !}$, а характеристика сложности атаки на секретный автоморфизм равна $\frac{r+1}{t_{4} \cdot t_{6}}$. Это дает возможность выбирать элементы $(\sigma, \eta)$ из групп Aut $K$ и Aut $L$ меньших порядков, но с большими централизаторами. Важную роль здесь играет параметр $r$. С его помощью можно сбалансировать нашу криптосистему, делая одинаково сложными атаки на $\varphi$ и $\psi, \chi_{1}, \ldots, \chi_{r}$.

Авторы благодарны М. М. Глухову за внимание к работе и С. В. Полину за замечания, способствующие улучшению изложения.

\section{Список литературы}

1. Росошек С. К. Криптосистемы групповых колец. - Вестник Томского гос. ун-та, 2003, № 6, c. 57-62.

2. Ламбек И. Кольца и модули. - М.: Факториал Пресс, 2005, с. 283.

3. Белоусов В. Д. Основы теории квазигрупп и луп. - М.: Наука, 1967, с. 223.

4. Smith J.D. H. Representation Theory of Infinite Groups and Finite Quasigroups. Montreal: Univ. Montreal, 1986.

5. The GAP Group. GAP - Groups, Algorithms and Programming. Ver. 4.4.9, 2006. http://www.gap-system.org.

6. Vojtechovsky P. Loops - a GAP package. Version 1.4.0, 2007. - ftp://ftp.gapsystem.org/pub/gap/gap4/win.zip/packages/loops-1.4.0-win.zip.

7. Goodaire E. G. Advances in loop rings and their loops. - Quasigroups and relat. syst., 2007, v. 15, № 1, p. 1-18. 